\title{
Effect of the viral protease on the dynamics of bacteriophage HK97 maturation intermediates characterized by variance analysis of cryo EM particle ensembles

\author{
Yunye Gong \\ Cornell University \\ Ithaca, NY USA \\ yg326@cornell.edu \\ David Veesler \\ La Jolla, CA USA \\ Present address: \\ Department of Biochemistry \\ University of Washington \\ dveesler@uw.edu \\ Peter C. Doerschuk
} \\ Electrical and Computer Engineering \\ Integrative Structural and Computational Biology \\ The Scripps Research Institute \\ Seattle, WA USA
}

Biomedical and Electrical and Computer Engineering

Cornell University

Ithaca, NY USA

pd83@cornell.edu

Corresponding author:

Phillips Hall Room 305

Cornell University

Ithaca, NY 14853 USA

$607-255-2152$ 


\title{
John E. Johnson \\ Integrative Structural and Computational Biology \\ The Scripps Research Institute \\ La Jolla, CA USA \\ jackj@scripps.edu
}

December 22, 2015

\begin{abstract}
Cryo EM structures of maturation-intermediate Prohead I of bacteriophage HK97 with $\left(\mathrm{PhI}^{\mathrm{Pro}+}\right)$ and without $\left(\mathrm{PhI}^{\mathrm{Pro}-}\right)$ the viral protease packaged have been reported (Veesler et al., Structure 22:230, 2014). In spite of $\mathrm{PhI}^{\mathrm{Pro}+}$ containing an additional $\sim 100 \times 24 \mathrm{kD}$ of protein, the two structures appeared identical although the two particles have substantially different biochemical properties, e.g., $\mathrm{PhI}^{\mathrm{Pro}-}$ is less stable to disassembly conditions such as urea. Here the same cryo EM images are used to characterize the spatial heterogeneity of the particles at $17 \AA$ resolution by variance analysis and show that $\mathrm{PhI}{ }^{\mathrm{Pro}-}$ has roughly twice the standard deviation of $\mathrm{PhI}^{\mathrm{Pro}+}$. Furthermore, the greatest differences in standard deviation are present in the region where the $\delta$-domain, not seen in x-ray crystallographic structures or fully seen in cryo EM, is expected to be located. Thus presence of the protease appears to stabilize the $\delta$-domain which the protease will eventually digest.

Key words: cryo electron microscopy; virus maturation; Hong Kong 97 bacteriophage; viral protease.
\end{abstract}

\section{Introduction}

Virus particle maturation is a well-orchestrated series of transitions in which the particle goes from a non infectious provirion to a mature, infectious virus particle. Double stranded (ds) 
DNA bacteriophage maturation is characterized by an elaborate sequence of intermediates and a number of these have been stabilized and isolated for bacteriophage HK97 by employing virus-like particles (VLPs). Co-expression of the capsid protein and a virus encoded protease in E. coli leads to a stable Prohead II intermediate that is the substrate for dsDNA packaging. This particle results from assembly of 420 subunits of identical sequence that form a $T=7 l$ quasi-equivalent capsid with about 100 copies of the viral protease packaged. The protease is activated upon completion of particle assembly and digests 103 amino acids ( $\delta$-domain) from the $\mathrm{N}$-termini of the capsid proteins and then the protease auto-digests with all of the polypeptide fragments diffusing from the particle through holes in the capsid. Prohead I, the particle containing full-length capsid proteins and the viral protease molecules, can be isolated if a mutation is introduced in the protease to make it non functional. Prohead I, without protease, can also be made by expressing only the capsid protein. Previously cryo EM structures were reported (Veesler et al., 2014) of the Prohead I with protease $\left(\mathrm{PhI}^{\mathrm{Pro}+}\right)$ and without protease $\left(\mathrm{PhI}^{\mathrm{Pro}-}\right)$ and shown to be closely similar with the protease density largely disordered. Hydrogen deuterium exchange experiments in the same study demonstrated that the $\delta$-domain residues were largely protected in $\mathrm{PhI}^{\mathrm{Pro}+}$ compared to $\mathrm{PhI}^{\mathrm{Pro}-}$ indicating that the protease bound to its own substrate prior to digestion. Biochemical experiments showed that $\mathrm{PhI}^{\mathrm{Pro}+}$ particles were more resistant to urea and other disassembly conditions compared to $\mathrm{PhI}^{\mathrm{Pro}-}$ suggesting its overall greater stability.

Previously we used the analysis of cryo EM particle ensembles to characterize particle dynamics of maturation intermediates of the RNA virus N $\omega \mathrm{V}$ (Tang et al., 2014; Wang et al., 2013). We showed that particles in which a maturation dependent auto-catalytic cleavage was inhibited by mutation showed much greater variance in the ensemble of particles than mature particles that had undergone auto-cleavage. The approach treats each particle of the ensemble as a single instance that is preserved at the moment of freezing. The continuous heterogeneity displayed by the ensemble is characterized by treating the Fourier series coef- 
ficients of the resultant reconstruction, not as individual numbers, but as Gaussian random variables. The coefficients and their variance are computed with a maximum likelihood (ML) algorithm thereby characterizing the heterogeneity of the entire ensemble. Employing this method results in reconstructions that are closely similar to those computed with traditional methods, but it is also possible to plot the variance of the reconstruction at each voxel providing a powerful method to examine particle heterogeneity based on first principles.

There are several alternative approaches for characterizing heterogeneity. With enough computing, many systems can compute the mean function and second moment function ${ }^{1}$ at each voxel in the 3-D reconstruction by resampling (Penczek and Spahn, 2011; Spahn and Penczek, 2009; Zhang et al., 2008; Penczek et al., 2006; Simonetti et al., 2008). Resampling is the process of randomly selecting images from the image stack and using the randomly selected images to compute a reconstruction. Repeating this process many times results in many reconstructions and the mean and second moment at each voxel can then be computed by standard statistics. As we have discussed (Wang et al., 2013, Section 3.2.6), an advantage of resampling is that very little must be assumed about the probability density functions, perhaps just the existence of convergence theorems for averages. However, the assumptions we use (which are described in detail in Section A.4 in the Supplemental material) have a long history (dating back to at least 1984 (Redner and Walker, 1984)) in the pattern recognition and machine learning communities as assumptions that are still useful even if there is no underlying physical model to motivate them. It is difficult to discuss the relative requirements on the number of images and computation because they depend on the reconstruction algorithm that is used in the resampling approach. Other investigators

\footnotetext{
${ }^{1}$ Let $\rho(\mathbf{x})$ be the electron scattering intensity at location $\mathbf{x}$. The mean function (denoted by $\bar{\rho}(\mathbf{x})$ ), which is the structure, at location $\mathbf{x}$ is the average over all of the particles of $\rho(\mathbf{x})$. The covariance function, which characterizes the heterogeneity of the ensemble of particles in a particular class, is the average of $\left[\rho\left(\mathbf{x}_{1}\right)-\bar{\rho}\left(\mathbf{x}_{1}\right)\right]\left[\rho\left(\mathbf{x}_{2}\right)-\bar{\rho}\left(\mathbf{x}_{2}\right)\right]$ where $\mathbf{x}_{1}$ and $\mathbf{x}_{2}$ are not necessarily equal. The second moment function is the average of $[\rho(\mathbf{x})]^{2}$ and the variance function is the average of $[\rho(\mathbf{x})-\bar{\rho}(\mathbf{x})]^{2}$. The second moment and variance functions can be computed from the covariance function but the covariance function cannot be computed from the second moment or the variance function.
} 
estimate the covariance of the voxel values from the sample covariance of the images (Liao et al., 2015; Liao and Frank, 2010; Katsevich et al., 2015). Resolution is limited, e.g., $16 \times 16 \times 16$ in Liao et al. (2015), and it is not clear how to account for the measurement noise that contaminates experimental images. Other investigators base their analysis on having an atomic or pseudo-atomic structure from which normal modes can be computed (Jin et al., 2014). While such structures are increasingly available from cryo EM itself, this type of approach is less directly based on the cryo EM images than the approach used in this paper, it is not clear how to account for the measurement noise, and the computations are extensive.

Given the availability of cryo EM data sets for HK97 $\mathrm{PhI}{ }^{\mathrm{Pro}+}$ and $\mathrm{PhI}^{\mathrm{Pro}-}$ and the previously characterized difference in biochemical stability we applied this ML Gaussian mixture approach to look for differences in particle heterogeneity of these ensembles that would be a structural signature of the difference in particle stability. The results were striking, with an overall standard deviation (the square root of variance) of the $\mathrm{PhI}^{\mathrm{Pro}-}$ ensemble being approximately twice that of $\mathrm{PhI}^{\mathrm{Pro}+}$. The standard deviation was then mapped on to the atomic models fitted into the cryo EM density, allowing an explicit description of how the standard deviation was distributed over the 7 quasi-equivalent molecules in the two structures.

\section{Mathematical model and computational methods}

We characterize the heterogeneity of the particles by improved versions of the mathematical model and computational methods described in Wang et al. (2013). In the following paragraphs we sketch the approach; more detail is included in the Supplemental material.

The electron scattering intensity of a particle is described as a Fourier series. Because the particles are heterogeneous, the Fourier coefficients can differ from particle to particle. Rather than attempt to estimate the coefficients for each particle, which is difficult from a 
single cryo EM image of each particle, we treat the coefficients as random variables rather than as unknown numbers and estimate the probability density function (pdf) of the random variables. We assume that the particles come from one of a small number of discrete classes and that within each class the pdf is Gaussian, which implies that the pdf is completely determined by the mean vector and the covariance matrix of the vector of Fourier coefficients for that class. Therefore, the computation to characterize the heterogeneity is an estimator to determine the mean vectors and covariance matrices from the cryo EM images. In the remainder of this paper, we only describe and give equations for the one-class case (Zheng et al., 2012).

Since the transformation from Fourier coefficients to the image is linear, there is a matrix $L$, which depends on the projection direction (denoted by $\theta_{i}$ for the $i$ th image), such that the noise-free image of the $i$ th particle with the pixel values arrayed as a vector $\check{y}_{i}$ is $\check{y}_{i}=L\left(\theta_{i}\right) c_{i}$ where $c_{i}$ are the coefficients for the $i$ th particle arrayed as a vector. The image is corrupted by noise (denoted by $w_{i}$ for the $i$ th image) which we describe as additive zero-mean Gaussian white noise with variance $\lambda^{2}$. Therefore the measured image is

$$
y_{i}=L\left(\theta_{i}\right) c_{i}+w_{i} .
$$

The assumption that both the coefficients $c_{i}$ and the noise $w_{i}$ are Gaussian leads to substantial simplifications in the algorithms because linear combinations of Gaussian random variables are themselves Gaussian random variables.

We estimate the mean vector $\bar{c}$ and the covariance matrix $V$ of the coefficients $c_{i}$ from the cryo EM images by a maximum likelihood (ML) estimator. The estimator is computed by an expectation-maximization (E-M) algorithm. The so-called nuisance parameters in an E-M algorithm are parameters whose values are not known but which, if their values were known, would greatly simplify the calculation of the ML estimate. The nuisance parameters 
that we use are the projection orientation. It is required to provide a pdf on the nuisance parameters and a pdf that is uniform over all orientations has been sufficient for spherical viruses. Each iteration of the E-M algorithm requires an expectation over the nuisance parameters followed by maximizing the result of the expectation with respect to the unknown parameters in order to yield the next value of the unknown parameters. The expectation must be computed numerically and is the primary computational expense of the algorithm. As is fully described in the Supplemental material (Section C.3), the computation of the expectation has been accelerated by not including terms where the pdf is negligible. This can also be interpreted as exploiting a priori knowledge (Section C.4). In addition, parts of the numerical linear algebra have been reorganized (Section C.1). The maximization for the mean vector $\bar{c}$ can be determined symbolically and therefore only one set of expectation integrals is required. However, the maximization for the covariance matrix $V$ is computed by an iterative numerical algorithm. Therefore multiple sets of expectation integrals must be computed, one set for each iteration. In order to minimize the number of iterations, a more efficient maximization algorithm is used (Supplemental material Section C.2). These changes have decreased the computation time by approximately a factor of 2-3.

Either hand for a structure is equally consistent with a set of cryo EM images which include only one image per particle. For HK97, the hand can be determined at low resolution because it is known that the surface lattice of the particle is $T=7 l$ not $T=7 d$. For the Fourier series basis functions used in this paper, a simple algorithm exists for changing the hand of the structure (Supplemental material Section E.1).

The two datasets $\mathrm{PhI}^{\mathrm{Pro}+}$ and $\mathrm{PhI}^{\mathrm{Pro}-}$ were recorded separately and not scaled together. Therefore, in order to compare the characterization of heterogeneity for the two datasets, we scale the two structures by least squares (Supplemental material Section E.2).

We compute resolution from Fourier Shell Correlation between structures which can be evaluated either by standard software applied to the cubes (e.g., EMAN2 (Tang et al., 2007)) 
or by formulas based on the Fourier series basis functions used in this paper (Supplemental material Section E.3). The resolution is not limited by the number or quality of the cryo EM images but rather by the limitations of our computer hardware (memory size and computational speed) which limit the number of Fourier coefficients that we can use.

From the mean vector and covariance matrix of the Fourier coefficients we can compute the mean (which is the structure) and the covariance (which is the characterization of the heterogeneity) of the electron scattering intensity. Because the Fourier coefficients are described as Gaussian and the transformation from coefficients to intensity is linear, the electron scattering intensity is Gaussian and is completely described by its mean and covariance functions. The mean is the average of the intensity at some 3-D location in real space and is denoted by $\bar{\rho}(\mathbf{x})$ where $\mathbf{x}$ is the location. The mean is the equivalent of the structure from a standard algorithm. Let $\rho_{i}(\mathbf{x})$ be the intensity of the $i$ th particle and let $\delta_{i}(\mathbf{x})=\rho_{i}(\mathbf{x})-\bar{\rho}(\mathbf{x})$ be the deviation of the intensity of the $i$ th particle from the mean. The covariance is the average of the product of the deviation at two possibly different locations, i.e., the average of $\delta_{i}\left(\mathbf{x}_{1}\right) \delta_{i}\left(\mathbf{x}_{2}\right)$, and is denoted by $C_{\rho}\left(\mathbf{x}_{1}, \mathbf{x}_{2}\right)$. The covariance is the fundamental characterization of the heterogeneity of the particle. We specialize the covariance function to the variance function which is defined by $v(\mathbf{x})=C_{\rho}(\mathbf{x}, \mathbf{x})$ and to the square root of the variance function which is $s(\mathbf{x})=\sqrt{v(\mathbf{x})}$. In order to set notation, the original Fourier series for the electron scattering intensity of the $i$ th particle is

$$
\rho_{i}(\mathbf{x})=\sum_{\alpha} c_{i, \alpha} \phi_{\alpha}(\mathbf{x})
$$

where $\phi_{\alpha}(\mathbf{x})$ is the $\alpha$ th basis function evaluated at location $\mathbf{x}$. Then the mean function for the electron scattering intensity is (Wang et al., 2013, Eq. 30)

$$
\bar{\rho}(\mathbf{x})=\sum_{\alpha} \bar{c}_{\alpha} \phi_{\alpha}(\mathbf{x})
$$


which is a three dimensional cube just like the structure from a standard algorithm. In addition, the covariance function for the electron scattering intensity is (Zheng et al., 2012, Eq. 18)

$$
C_{\rho}\left(\mathbf{x}_{1}, \mathbf{x}_{2}\right)=\sum_{\alpha_{1}} \sum_{\alpha_{2}} V_{\alpha_{1}, \alpha_{2}} \phi_{\alpha_{1}}\left(\mathbf{x}_{1}\right) \phi_{\alpha_{2}}\left(\mathbf{x}_{2}\right)
$$

which is a six dimensional cube. Finally, the variance function is (Wang et al., 2013, Eq. 32)

$$
v(\mathbf{x})=\sum_{\alpha_{1}} \sum_{\alpha_{2}} V_{\alpha_{1}, \alpha_{2}} \phi_{\alpha_{1}}(\mathbf{x}) \phi_{\alpha_{2}}(\mathbf{x})
$$

which is a three dimensional cube just like the structure from a standard algorithm. Both $C_{\rho}\left(\mathbf{x}_{1}, \mathbf{x}_{2}\right)$ (Eq. 4) and $v(\mathbf{x})($ Eq. 5) can be computed for any covariance matrix $V$ with elements $V_{\alpha_{1}, \alpha_{2}}$ including simplifying cases such as assuming that $V$ is diagonal. While $C_{\rho}\left(\mathbf{x}_{1}, \mathbf{x}_{2}\right)$ contains more information than $v(\mathbf{x})$, much of our understanding of the particles' heterogeneity is based on $v(\mathbf{x})$ because of the difficulty of understanding a six dimensional cube. Most commonly it is the standard deviation that is displayed, rather than the variance, because the standard deviation, the mean, and a structure all share the same units. In the numerical results of Section 4 it is assumed that $V$ is a diagonal matrix which reduces the size of the optimization problem. In particular, if $N$ Fourier coefficients are used then there are $N$ unknown numbers to describe the mean vector and $N(N+1) / 2$ unknown numbers to describe the full covariance matrix which are reduced to $N$ and $N$ when $V$ is diagonal and this reduction in the number of unknowns is necessary due to limitations of our computer hardware (memory size and computational speed).

To describe the variance we use a variety of visualization tools (Supplemental material Section E.4): (1) Histograms of the standard deviation $(s(\mathbf{x}))$. (2) Images of the surface of the structure $(\bar{\rho}(\mathbf{x}))$ colored by the standard deviation $(s(\mathbf{x}))$ via USCF Chimera (Pettersen et al., 2004). (3) Plots of the square root of the spherical average of the variance. (4) When 
atomic resolution structures are available, ribbon diagrams colored by standard deviation values. In many instances we display the difference of standard deviation between the $\mathrm{PhI}{ }^{\mathrm{Pro}+}$ and $\mathrm{PhI}^{\mathrm{Pro}-}$ structures.

\section{Materials and methods}

Two $\mathrm{PhI}^{\mathrm{Pro}+}$ data sets were created by random extraction of non-overlapping sets of 1200 boxed images from the larger image stack of Veesler et al. (2014) which is CTF corrected. Because the effect of the CTF is already removed, we can decrease the size of the boxed image (Supplemental material Section D). The same process was applied to the $\mathrm{PhI}{ }^{\mathrm{Pro}-}$ image stack of Veesler et al. (2014), which is also CTF corrected, resulting in a total of four stacks of 1200 images each. The boxed images are $352 \times 352$ pixels in dimension with a

sampling interval of $2.76 \AA$ /pixel. The boxed images were truncated to $200 \times 200$ pixels which is a tight fit around the particle. Four different structures using 1060 basis functions (all Fourier series basis functions with $l \leq 55, n$, and $p \leq 20$, Supplemental material Section A especially Eq. 3) were computed starting from an initial condition where no heterogeneity was allowed (i.e., $V=0$ ). The computations were done using a desktop $\mathrm{PC}$ with a program written in Matlab and took 1-2 days per reconstruction. Computation time and memory requirements limit the number of images used (the image collections of Veesler et al. (2014) are much larger than 1200 images) and number of coefficients used (which puts a bound on resolution that is independent of the bound due to the number and quality of the images).

\section{Results}

In this section we present results on the quality of the numerical approximation that we have introduced in order to decrease computation time, the resolution of the results, the nature 
of the structures of $\mathrm{PhI}^{\mathrm{Pro}+}$ and $\mathrm{PhI}^{\mathrm{Pro}-}$, and the characterization of the heterogeneity of $\mathrm{PhI}^{\mathrm{Pro}+}$ and $\mathrm{PhI}^{\mathrm{Pro}-}$. A simplified example (a 1-D example where the relationship between the 1-D particle and the 1-D image lacks a projection operation, the CTF is unity at all spatial frequencies, and the location of the origin in the 1-D image is known) is presented in Supplemental material Section F Figure 2.

In order to measure the effect of approximating the expectation integrals in the E-M algorithm by not including terms where the probability density function is negligible (Section 2 and Supplemental material Section C.3), the calculations have been performed both with and without the approximation on one of the $\mathrm{PhI}^{\mathrm{Pro}+}$ cryo EM data sets. The result is that the Fourier Shell Correlation (FSC) plots between the two calculations are above 0.9 for more than the range $k \leq 0.06 \AA^{-1}$ and therefore the structures are essentially identical for more than the range $k \leq 0.06 \AA^{-1}$. Higher spatial frequencies are not relevant because computer hardware limitations (memory size and computational speed) limit the number of Fourier coefficients that can be used so the mathematical model does not represent spatial fluctuations at higher spatial frequencies.

Figure 1 shows the Fourier Shell Correlation (FSC) plots for the $\mathrm{PhI}^{\mathrm{Pro}+}$ and $\mathrm{PhI}^{\mathrm{Pro}-}$ cryo EM data sets. In the case of $\mathrm{PhI}^{\mathrm{Pro}-}$, the resolution is determined by the fact that the FSC curve crosses the 0.5 level at approximately $0.057 \AA^{-1}$ giving a resolution of $17.5 \AA$. In the case of PhI ${ }^{\mathrm{Pro}+}$, the FSC curve never drops below 0.5. However, due to computer hardware limitations which restrict the number of Fourier coefficients that can be used, the energy in both structures (the denominators of the FSC calculation) have dropped below $10^{-4}$ times their peak values by $0.06 \AA^{-1}$ so that higher scattering angles are not relevant and a resolution of $0.06 \AA^{-1}$ or $16.7 \AA$ is achieved. When an FSC curve is computed between the two data sets it is essentially identical to the FSC curves of the individual data sets indicating that in spite of a difference in mass of roughly $240 \mathrm{kD}(\sim 100$ copies of the viral protease with a mass of $24 \mathrm{kD}$ in $\mathrm{PhI}^{\mathrm{Pro}+}$ that are absent in $\mathrm{PhI}^{\mathrm{Pro}-}$ ), the structures are 
identical to $17 \AA$ resolution. This was the same conclusion reached by Veesler et al. (2014) when the electron scattering density of the two maps were compared and difference maps computed in an effort to visualize the protease density. The implication is that the protease is sufficiently disordered relative to the icosahedral structure of the particles that there is no reinforced density when icosahedral symmetry is applied. That the two structures are essentially identical is the motivation for the identical means in the two particles of the simplified example (Supplemental material Section F Figure 2). As is described in Supplemental material Section E.2, the virtually identical $17 \AA$ electron scattering densities were carefully scaled together and the corresponding scaling was applied to the variance cubes.

The difference in the data sets was immediately obvious when the distribution of variance was computed for all voxels within the shell of density that accommodated the atomic model of residues 103 to 351 in the viral capsid protein. Figure 2 shows that $98 \%$ of the voxels in $\mathrm{PhI}^{\mathrm{Pro}+}$ have a standard deviation of less than $0.6 \times 10^{-3}$, while all of the voxels in $\mathrm{PhI}^{\mathrm{Pro}-}$ have a standard deviation greater than $0.6 \times 10^{-3}$. That the two heterogeneity characterizations are quite different is the motivation for the different variances in the two particles of the simplified example (Supplemental material Section F Figure 2).

A more dramatic difference is seen in Figure 3(a) where the square root of the spherically averaged variance is plotted as a function of radius for both data sets, in which case it is seen that the heterogeneity overall for $\mathrm{PhI}^{\mathrm{Pro}-}$ is roughly twice that of $\mathrm{PhI}^{\mathrm{Pro}+}$ and even greater at lower radii where the protease is thought to bind. Figure 3(b) shows the fractional difference in heterogeneity for the two data sets $\left(\left(\mathrm{PhI}^{\mathrm{Pro}-}-\mathrm{PhI}^{\mathrm{Pro}+}\right) / \mathrm{PhI}^{\mathrm{Pro}+}\right)$, emphasizing that the heterogeneity for $\mathrm{PhI}^{\mathrm{Pro}-}$ is always much higher than the heterogeneity of $\mathrm{PhI}^{\mathrm{Pro}+}$.

It is possible to have structures of a particular resolution with quite different variance cubes because the mean of the Fourier coefficients determines the structure and the covariance of the Fourier coefficients determines the heterogeneity and the mean and covariance 
are independent, This is the case for $\mathrm{PhI}^{\mathrm{Pro}+}$ and $\mathrm{PhI}^{\mathrm{Pro}-}$ where the resolutions are both approximately $17 \AA$ resolution but the variance cubes (spherically averaged in Figure 3 followed by taking the square root) differ by a factor of two.

Figure 4 presents the color-coded surface heterogeneity for both data sets. In the top row different color bars were chosen for $\mathrm{PhI}^{\mathrm{Pro}+}$ and $\mathrm{PhI}^{\mathrm{Pro}-}$ to emphasize the variation that occurs across the surface of each structure. In the bottom row the same color bar was chosen for both data sets to emphasize the dramatically lower overall surface heterogeneity in $\mathrm{PhI}^{\mathrm{Pro}+}$ compared to $\mathrm{PhI}^{\mathrm{Pro}-}$. The feature emphasized by these images is that the $\mathrm{PhI}{ }^{\mathrm{Pro}-}$ particle has substantially higher heterogeneity than the $\mathrm{PhI}^{\mathrm{Pro}+}$ particle.

Figure 5 shows ribbon diagrams colored by the heterogeneity of $\mathrm{PhI}^{\mathrm{Pro}-}$ minus the heterogeneity of $\mathrm{PhI}^{\mathrm{Pro}+}$. In the side view (Figure 5(b)), the alpha helices extending downward in the image, corresponding to extending inward in the particle, are the $20 \%$ of the $\delta$-domain based on the pseudo-atomic coordinates from the cryo EM structure (Veesler et al., 2014). All of the $\delta$-domain is disordered in the x-ray crystallography structure of Prohead-I (Huang et al., 2011) (3p8q). The greatest spatial regions of large differences in variance in Figure 5(b) are the $\delta$-domains which occupy the radial region where the $\sim 100$ copies of the viral protease are expected to be located.

Figure 6 shows surface and cross section plots where the colors are determined by the same difference visualized in Figure 5. This type of visualization can be computed without any of the atomic coordinates needed for the ribbon diagrams of Figure 5. Again, the greatest regions of large differences are the $\delta$-domains.

Finally, Figure 7 plots the difference as a function of amino acid residue number. This plot quantitatively shows the heterogeneity information from the ribbon diagrams of Figure 5 but lacks the geometric information from Figure 5. The $\delta$-domain is residues $1-103$. In addition to showing elevated differences in each $\delta$-domain this plot emphasizes that most of each $\delta$-domain is not visualized (the "Residue number" axis does not start at Residue Number 1) 
in either x-ray crystallography or cryo EM structures.

\section{Discussion}

The data presented above describe an unusual situation in structural biology. The structures determined are indistinguishable at the resolution of the reconstructions of this paper, but the variance of the density is dramatically different. The simplified example (Supplemental material Section F Figure 2) shows the same situation. Instances of $\mathrm{PhI}^{\mathrm{Pro}-}$ display exceptional heterogeneity over the ensemble of particles processed when compared to $\mathrm{PhI}^{\mathrm{Pro}+}$. The method for extracting the variation in a statistically robust manner is novel and without it the results presented could not have been obtained.

In this paper the images are already classified, i.e., $\mathrm{PhI}^{\mathrm{Pro}+}$ or $\mathrm{PhI}^{\mathrm{Pro}-}$, and so we do no clustering. However, the approach can include clustering. In particular, ensembles of particles that differ in their structure, ensembles of particles that share the same structure but differ in their variance, and ensembles of particles that differ in both structure and variance can all be clustered. These types of calculations have been demonstrated in unpublished work based on simulated images. Many popular programs exist which can cluster particles that differ in their structure, but the ability to classify particles of the same structure but different variances is less common. Programs that classify based on structure can analyze images from heterogeneous particles of a single biological class, e.g., $\mathrm{PhI}^{\mathrm{Pro}+}$, by making multiple subclasses and computing a structure for each sub-class. Computing structures for each of many sub-classes is challenging because the sub-classes will not be highly distinct. A subclass structure would not exactly predict the images associated with that sub-class because there would be some residual particle heterogeneity within the sub-class. The approach of this paper concisely and robustly describes the continuous heterogeneity by computing a single structure and a variance map. In addition to its utility in clustering, analysis of the 
variance can provide much biological information, as is demonstrated in this paper.

Figure 3 shows both the square root of the spherical average of the variance as a function of radius and the portions of the subunit and the protease assumed to occupy the radial shells. The 3-D variance is described in Figure 6 and motivates significant insights concerning the effect of packaging the protease in $\mathrm{PhI}^{\mathrm{Pro}+}$. Previously it was shown with $\mathrm{HD} / \mathrm{X}$ analysis that regions of the $\delta$-domain were most protected in the presence of protease indicating that the protease bound with significant affinity to the portion of the capsid protein that it would digest. Figure 6 shows that the greatest difference in variance between $\mathrm{PhI}^{\mathrm{Pro}+}$ and $\mathrm{PhI}^{\mathrm{Pro}-}$ occurs in the inner radial region where the $\delta$-domain is assumed to exist. This large difference presumably results from the great decrease in heterogeneity imposed when the protease binds to the $\delta$-domain, thus implicitly localizing the protease to this region of the 3-D map.

The implications of such a result are noteworthy, in that there are two data sets that can only be distinguished by the variance or heterogeneity in the particle ensembles, not by the Fourier coefficients derived from the structure and used to compute the 3-D structure. The result is in agreement with the $\mathrm{HD} / \mathrm{X}$ studies that localized the protease to the $\delta$-domain and the biochemical studies that clearly revealed $\mathrm{PhI}^{\mathrm{Pro}+}$ was significantly more stable to disassembly than $\mathrm{PhI}^{\mathrm{Pro}-}$. In an earlier structural study of PhI (Huang et al., 2011) it was only possible to crystallize $\mathrm{PhI}^{\mathrm{Pro}+}$, presumably due to its greater homogeneity and stability.

The difference in heterogeneity between $\mathrm{PhI}^{\mathrm{Pro}-}$ and $\mathrm{PhI}^{\mathrm{Pro}+}$ is maximal in the region of the $\delta$-domain. However, a substantial difference in heterogeneity is present throughout the capsid, including surface regions distant from the $\delta$-domain and the expected location of the viral protease. This is also the case in N $\omega \mathrm{V}$ (Tang et al., 2014) where a focused difference in the particles (a point mutation) causes a spatially broader change in heterogeneity. In both cases, failure to be able to follow the maturation pathway toward a more stable infectious particle leads to a spatially broad increase in heterogeneity. 
A similar situation was documented biochemically, but not structurally, for Flock House Virus (FHV), a ssRNA insect virus. FHV subunits contain a C-terminal polypeptide (363$407 ; \gamma)$ that is auto-catalytically cleaved during particle maturation and was shown by crystallography to be partially ordered and to reside non-covalently associated with the interior of the ordered capsid protein (Fisher and Johnson, 1993). These peptides facilitate cellular entry and are transiently exposed in solution. They were detected by incubating particles in the presence of trypsin and showing that $\gamma$ is the first polypeptide digested. VLPs of FHV were made by expressing the capsid protein in a baculovirus expression system resulting in particles that are indistinguishable by crystallography from authentic FHV. The difference between the two particle types is the packaged genome. Authentic FHV packages primarily the viral genome while VLPs package cellular RNA (Routh et al., 2012). Exposure of the $\gamma$ peptides was shown to be 7 times more frequent in VLPs than in authentic virus with the protease protocol and yet the crystal structures of the VLPs and virions were identical (Bothner et al., 1999). We propose that a cryo EM experiment of the type described here would readily distinguish the particle types by their variance, while the lattice effects in the crystal structure quench this dynamic character, making it undetectable.

Extraction of variance from particle ensembles in the cryo EM experiment enables novel structural insights that may not be directly observable from the electron scattering density. Currently the computational demand of the calculation relative to the hardware of our computer (memory size and computational speed) limits its application to roughly the nanometer resolution range for icosahedral virus particles. We are currently extending the technology to helical and asymmetric objects as well as improving the efficiency of the code for higher resolution work. The latter will potentially provide roughly the equivalent of crystallographic temperature factors for individual residues in protein structures that are not constrained by lattice quenching. 


\section{Acknowledgements}

Y.G. and P.C.D. are grateful to NSF 1217867 for funding. 


\section{References}

B. Bothner, A. Schneemann, D. Marshall, V. Reddy, J. E. Johnson, and G. Siuzdak. Crystallographically identical virus capsids display different properties in solution. Nature Structural Biology, 6:114-116, 1999.

A. Fisher and J. E. Johnson. Ordered duplex RNA controls capsid architecture in an icosahedral animal virus. Nature, 361:176-179, 1993.

Rick K. Huang, Reza Khayat, Kelly K. Lee, Ilya Gertsman, Robert L. Duda, Roger W. Hendrix, and John E. Johnson. The Prohead-I structure of bacteriophage HK97: Implications for scaffold-mediated control of particle assembly and maturation. J. Molecular Biology, 408(3):541-554, 6 May 2011.

Qiyu Jin, Carlos Oscar S. Sorzano, José Miguel de la Rosa-Trevín, José Román BilbaoCastro, Rafael Núñez-Ramírez, Oscar Llorca, Florence Tama, and Slavica Jonić. Iterative elastic 3D-to-2D alignment method using normal modes for studying structural dynamics of large macromolecular complexes. Structure, 22(3):496 - 506, 2014. ISSN 0969-2126. doi: http://dx.doi.org/10.1016/j.str.2014.01.004. URL http://www . sciencedirect.com/science/article/pii/S0969212614000136.

E. Katsevich, A. Katsevich, and A. Singer. Covariance matrix estimation for the cryo-EM heterogeneity problem. SIAM Imaging Science, 8(1):126-185, 2015.

H. Y. Liao and J. Frank. Classification by bootstrapping in single particle methods. In Proceedings of the 2010 IEEE International Symposium on Biomedical Imaging, pages 169-172, 14 Apr. 2010.

Hstau Y. Liao, Yaser Hashem, and Joachim Frank. Efficient estimation of three-dimensional 
covariance and its application in the analysis of heterogeneous samples in cryo-electron microscopy. Structure, 23(6):1129-1137, 2 June 2015.

Marek Penczek, Pawel A.and Kimmel and Christian M. T. Spahn. Identifying conformational states of macromolecules by eigen-analysis of resampled cryo-EM images. Structure, 19: 1582-1590, 9 November 2011.

Pawel A. Penczek, Chao Yang, Joachim Frank, and Christian M. T. Spahn. Estimation of variance in single-particle reconstruction using the bootstrap technique. J. Struct. Biol., 154(2):168-183, 2006.

E. F. Pettersen, T. D. Goddard, C. C. Huang, G. S. Couch, D. M. Greenblatt, E. C. Meng, and T. E. Ferrin. UCSF Chimera-A visualization system for exploratory research and analysis. J. Comput. Chem., 25(13):1605-1612, 2004.

Richard A. Redner and Homer F. Walker. Mixture densities, maximum likelihood and the EM algorithm. SIAM Review, 26(2):195-239, April 1984.

Andrew Routh, Tatiana Domitrovic, and John E. Johnson. Host rnas, including transposons, are encapsidated by a eukaryotic single-stranded rna virus. Proceedings of the National Academy of Sciences, 109(6):1907-1912, 2012. doi: 10.1073/pnas.1116168109. URL http: //www.pnas.org/content/109/6/1907. abstract.

Angelita Simonetti, Stefano Marzi, Alexander G. Myasnikov, Attilio Fabbretti, Marat Yusupov, Claudio O. Gualerzi, and Bruno P. Klaholz. Structure of the 30s translation initiation complex. Nature, 455(7211):416-420, 18 Sep. 2008. doi: \{\url\{10.1038/ nature07192\}\}. URL \{\url\{UR-http://dx.doi.org/10.1038/nature07192\}\}.

Christian M. T. Spahn and Pawel A. Penczek. Exploring conformational modes of macro- 
molecular assemblies by multiparticle cryo-EM. Current Opinion in Structural Biology, 19:623-631, 2009.

G. Tang, L. Peng, P. R. Baldwin, Mann D. S., W. Jiang, I. Rees, and S. J. Ludtke. EMAN2: An extensible image processing suite for electron microscopy. J. Struct. Biol., 157(1): $38-46,2007$.

Jinghua Tang, Bradley M. Kearney, Qiu Wang, Peter C. Doerschuk, Timothy S. Baker, and John E. Johnson. Dynamic and geometric analyses of Nudaurelia capensis $\omega$ virus maturation reveal the energy landscape of particle transitions. J. Molecular Recognition, 27(4):230-237, 10 February 2014.

David Veesler, Reza Khayat, Srinath Krishnamurthy, Joost Snijder, Rick K. Huang, Albert J. R. Heck, Ganesh S. Anand, and John E. Johnson. Architecture of a dsDNA viral capsid in complex with its maturation protease. Structure, 22:230-237, 2 February 2014.

Qiu Wang, Tsutomu Matsui, Tatiana Domitrovic, Yili Zheng, Peter C. Doerschuk, and John E. Johnson. Dynamics in cryo EM reconstructions visualized with maximumlikelihood derived variance maps. J. Struct. Biol., 181(3):195-206, March 2013.

Wei Zhang, Marek Kimmel, Christian M. T. Spahn, and Pawel A. Penczek. Heterogenneity of large macromolecular complexes revealed by 3D cryo-EM variance analysis. Structure, 16:1770-1776, 2008.

Yili Zheng, Qiu Wang, and Peter C. Doerschuk. 3-D reconstruction of the statistics of heterogeneous objects from a collection of one projection image of each object. J. Opt. Soc. Am. A, 29(6):959-970, June 2012. 


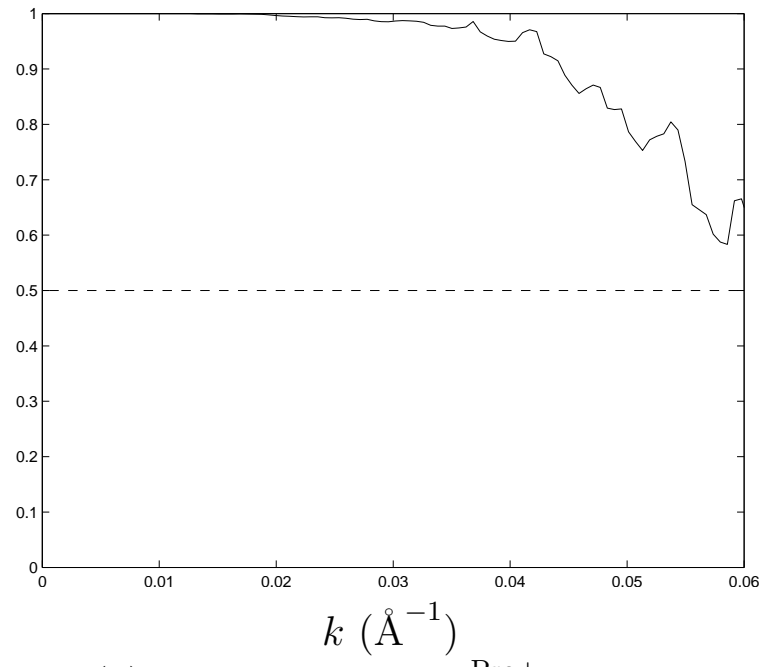

(a) Resolution of $\mathrm{PhI}^{\mathrm{Pro}+}$ by FSC

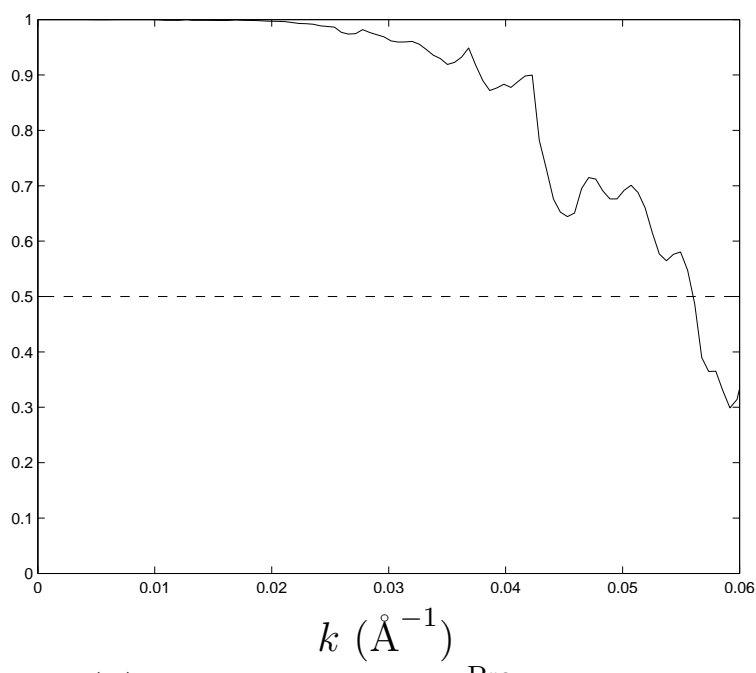

(b) Resolution of $\mathrm{PhI}{ }^{\mathrm{Pro}-}$ by FSC

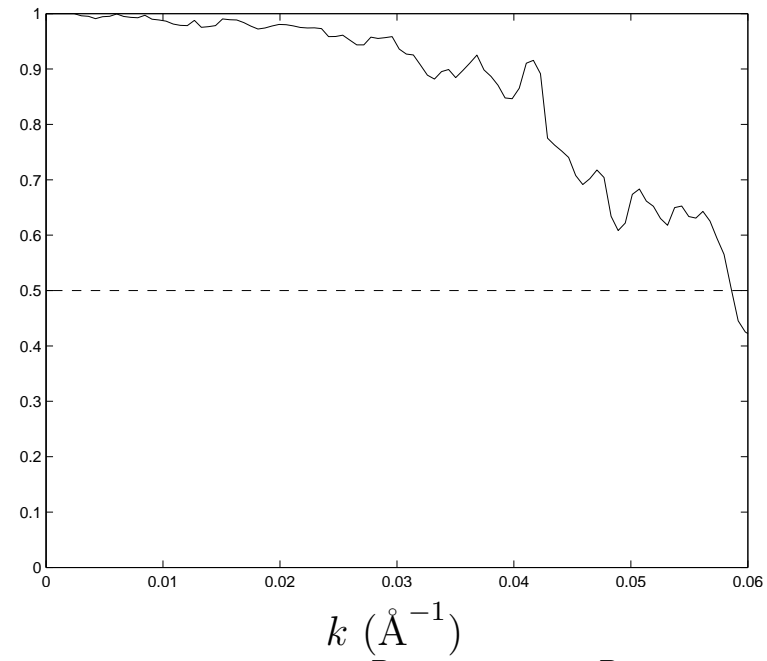

(c) Comparison of $\mathrm{PhI}^{\mathrm{Pro}+}$ and $\mathrm{PhI}^{\mathrm{Pro}-}$ by FSC

Figure 1: FSC plots for the mean electron scattering intensity which is the structure. The plots are truncated at $0.06 \AA^{-1}$ because the limited number of coefficients that are used in the mathematical model to describe the 3-D particle leads to a model which does not have significant scattering at higher scattering angles. The resolution of $\mathrm{PhI}^{\mathrm{Pro}+}$ and $\mathrm{PhI}^{\mathrm{Pro}-}$ are $16.7 \AA^{-1}$ and $17.5 \AA^{-1}$, respectively using an FSC cutoff of 0.5 . At the spatial resolution achieved by this number of coefficients in the mathematical model, there is no significant difference between the mean electron scattering intensities for $\mathrm{PhI}^{\mathrm{Pro}+}$ and $\mathrm{PhI}^{\mathrm{Pro}-}$. 


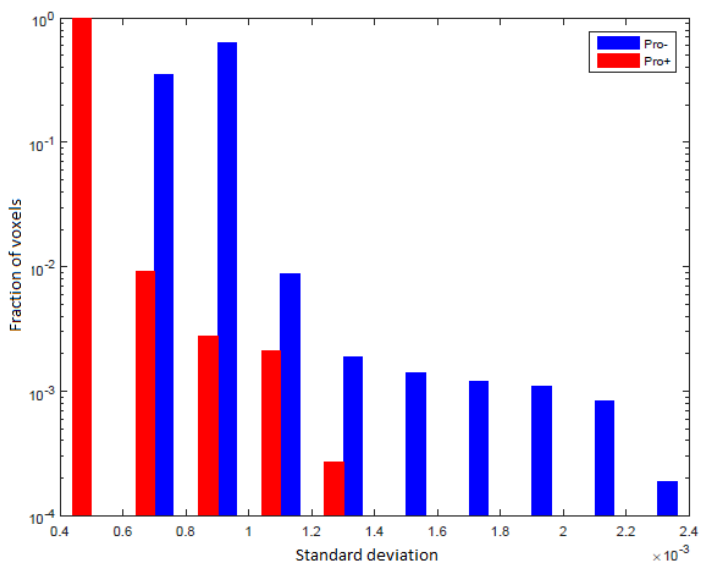

Figure 2: Heterogeneity is characterized by the standard deviation $s(\mathbf{x})$. Histograms of $s(\mathbf{x})$ for voxels in the capsid set $C$ defined in Supplemental material Section E.2 are displayed as fractions of the total number of voxels in $C$ on a logarithmic scale for both $\mathrm{PhI}^{\mathrm{Pro}+}$ and $\mathrm{PhI}^{\mathrm{Pro}-}$ and clearly demonstrate that the $\mathrm{PhI}^{\mathrm{Pro}-}$ structure has greater heterogeneity. The spatial distribution of heterogeneity is not visualized in this figure but is shown in detail in later figures. 


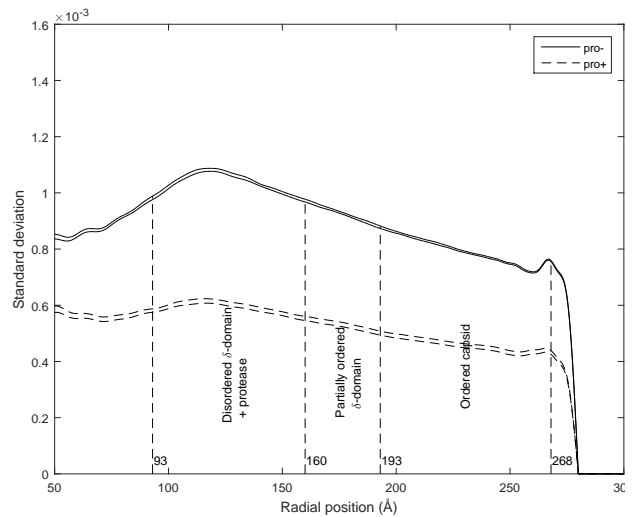

(a) The four curves show the spherical average, $\bar{s}(x)$, for Stacks 1 and 2 for $\mathrm{PhI}^{\mathrm{Pro}+}$ and for $\mathrm{PhI}^{\mathrm{Pro}-}$ (Supplemental material Section E.4 Item 3)

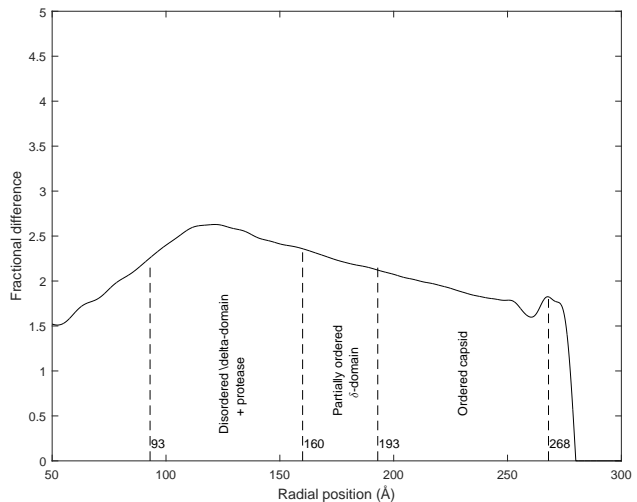

(b) After averaging the results for Stacks 1 and 2 for $\mathrm{PhI}^{\mathrm{Pro}+}$ and for $\mathrm{PhI}^{\mathrm{Pro}-}$, the one curve shows the fraction difference of the spherical average $(\bar{d}(x))$, specifically, $\quad\left(\mathrm{PhI}^{\mathrm{Pro}-}\right.$ $\mathrm{PhI}^{\mathrm{Pro}+}$ )/PhI ${ }^{\mathrm{Pro}+}$ (Supplemental material Section E.4 Item 4)

Figure 3: The radial distribution of heterogeneity as described by the square root of the spherical average of the variance. Several radii are marked. The largest and smallest radii of the atomic coordinates from the x-ray crystallography structure of Prohead-I (Huang et al., 2011) (3p8q) are $268 \AA$ and $193 \AA$. The smallest radius of the pseudo-atomic coordinates from the cryo EM structure (Veesler et al., 2014), which includes $20 \%$ of the $\delta$-domain, is $160 \AA$. The radius of the inner surface of the region of highest heterogeneity is $93 \AA$ (see also Figure 6(b)). The heterogeneity of $\mathrm{PhI}^{\mathrm{Pro}-}$ is substantially greater than the heterogeneity of $\mathrm{PhI}^{\mathrm{Pro}+}$ at all radii. The amount by which the heterogeneity of $\mathrm{PhI}^{\mathrm{Pro}-}$ exceeds the heterogeneity of $\mathrm{PhI}{ }^{\mathrm{Pro}+}$, which is most clearly visualized in the fractional plot, reaches a maximum at about a radius of $120 \AA$. This is potentially the radius at which the protease (which is nonfunctional in the $\mathrm{PhI}^{\mathrm{Pro}+}$ particles) associates with the $\delta$-domain and thereby reduces its heterogeneity. 


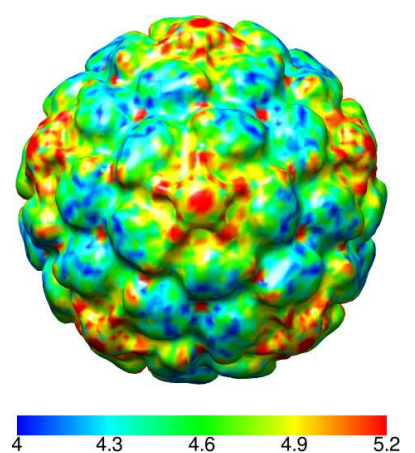

(a) $\mathrm{PhI}^{\mathrm{Pro}+}$

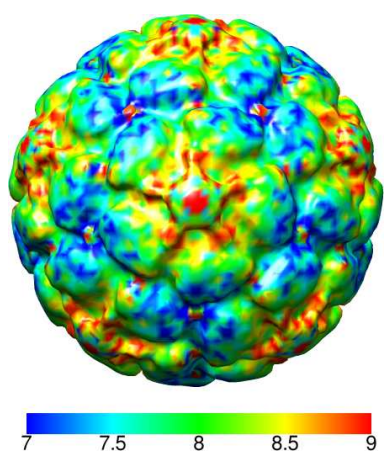

(b) $\mathrm{PhI}^{\mathrm{Pro}-}$

Different colorbars (markings scaled by $10^{4}$ ) for each visualization

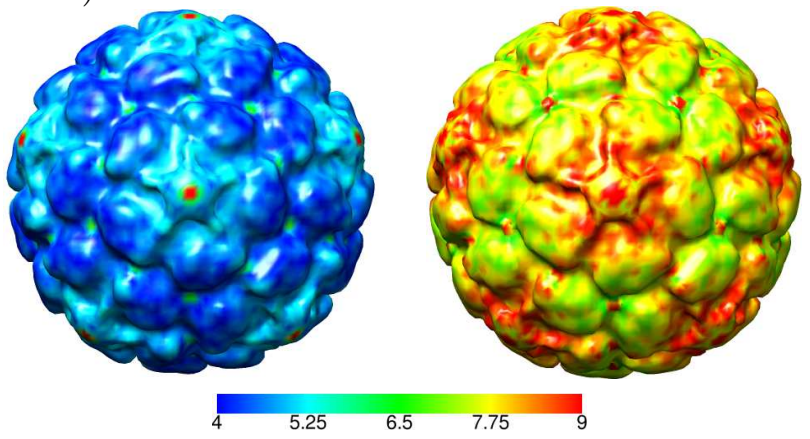

$\begin{array}{ll}\text { (c) } \mathrm{PhI}^{\mathrm{Pro}+} & \text { (d) } \mathrm{PhI}^{\mathrm{Pro}-}\end{array}$

Same colorbar (markings scaled by $10^{4}$ ) for both visualizations

Figure 4: Visualization of the heterogeneity by painting a surface of the mean electron scattering intensity (which is the structure) with colors determined by the standard deviation (which is the characterization of heterogeneity). The $\mathrm{PhI}^{\mathrm{Pro}-}$ structure has substantially greater heterogeneity than the $\mathrm{PhI}^{\mathrm{Pro}+}$ structure which is emphasized in the second row of visualizations where both visualizations share the same colorbar. Visualizations are computed by USCF Chimera (Pettersen et al., 2004). 


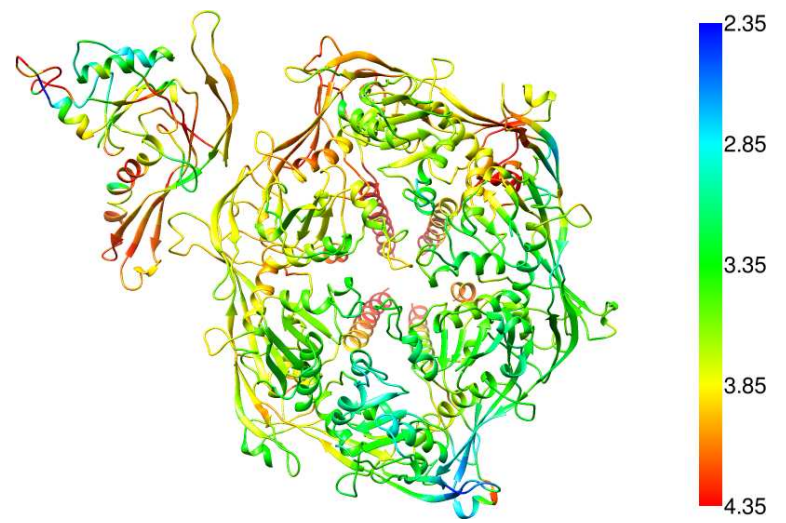

(a) From outside looking at the particle's center

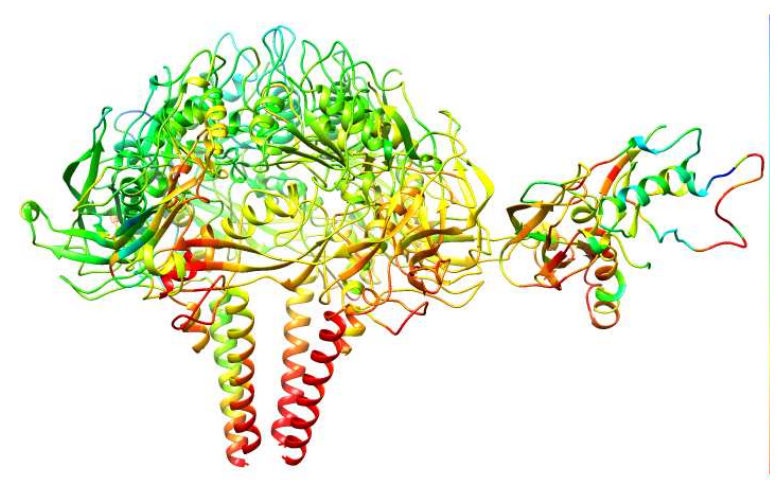

(b) From the side with the inner surface below.

Figure 5: Visualization of the heterogeneity by painting ribbon diagrams with colors determined by the standard deviation for $\mathrm{PhI}^{\mathrm{Pro}-}$ minus the standard deviation for $\mathrm{PhI}^{\mathrm{Pro}+}$ (Supplemental material Section E.4 Item 6). In the side view the $\delta$-domains from Veesler et al. (2014) are seen extending inward toward the center of the particle and have much larger heterogeneity in $\mathrm{PhI}^{\mathrm{Pro}-}$ than in $\mathrm{PhI}^{\mathrm{Pro}+}$ as is shown by the red color. Panels (a) and (b) share the same colorbar. Visualizations are computed by USCF Chimera (Pettersen et al., 2004). 


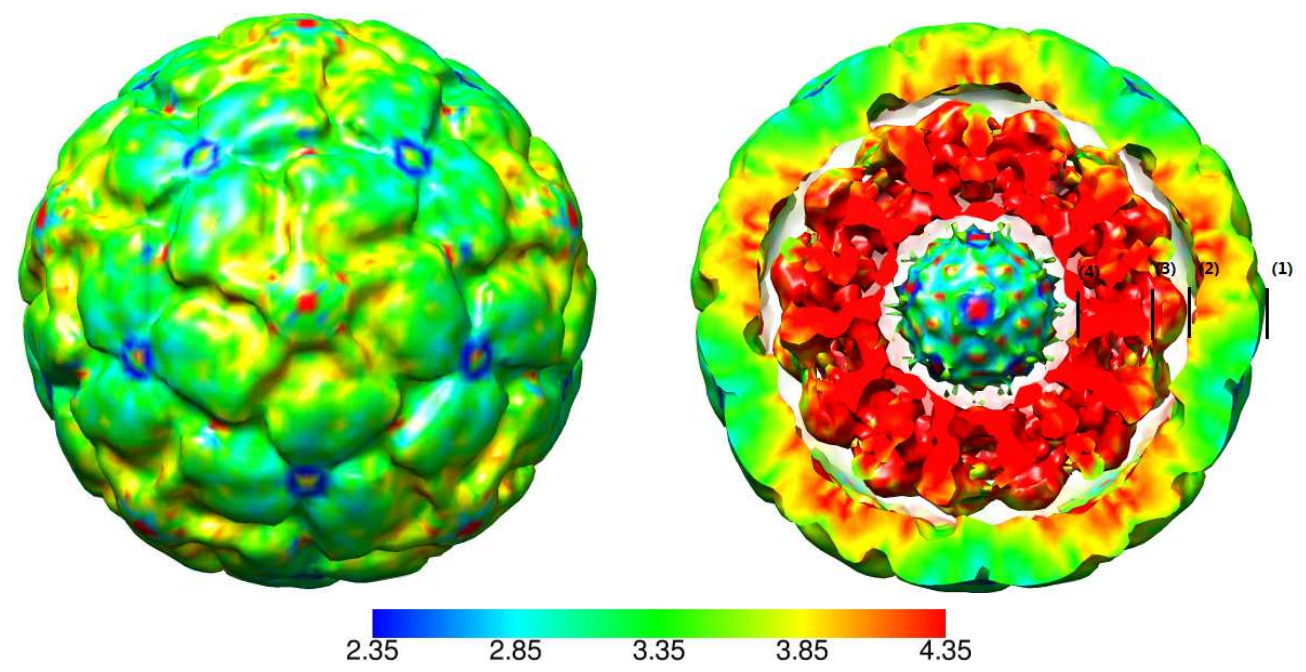

(a) Surface of the $\mathrm{PhI}^{\mathrm{Pro}-}$ struc(b) Central cross section perture pendicular to a 5 -fold symmetry axis of the $\mathrm{PhI}^{\mathrm{Pro}-}$ structure

Figure 6: The mean electron scattering intensity (which is the structure) of $\mathrm{PhI}^{\mathrm{Pro}-}$ colored by the standard deviation of $\mathrm{PhI}^{\mathrm{Pro}-}$ minus the standard deviation of $\mathrm{PhI}^{\mathrm{Pro}+}$. As is shown in Figures 1 and 4, the mean electron scattering intensities of $\mathrm{PhI}^{\mathrm{Pro}+}$ and $\mathrm{PhI}^{\mathrm{Pro}-}$ are very similar. The four lines on the positive horizontal axis in Panel (b) labeled "(1)"-"(4)" are

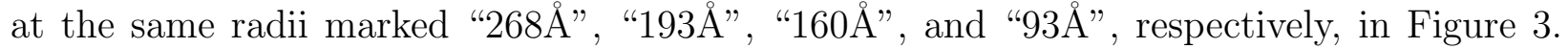
The radii of the x-ray crystallography atomic coordinates (Huang et al., 2011) lie between labels (1) and (2), the radii of the additional pseudo-atomic coordinates fit into the cryo EM map (Veesler et al., 2014) that represent approximately $20 \%$ of the $\delta$-domain lie between labels (2) and (3), and the largest values of the difference in the standard deviation of $\mathrm{PhI}^{\mathrm{Pro}-}$ minus the standard deviation of $\mathrm{PhI}^{\mathrm{Pro}+}$ lie between labels (3) and (4). Panels (a) and (b) share the same colorbar (markings scaled by $10^{4}$ ). Visualizations are computed by USCF Chimera (Pettersen et al., 2004). 


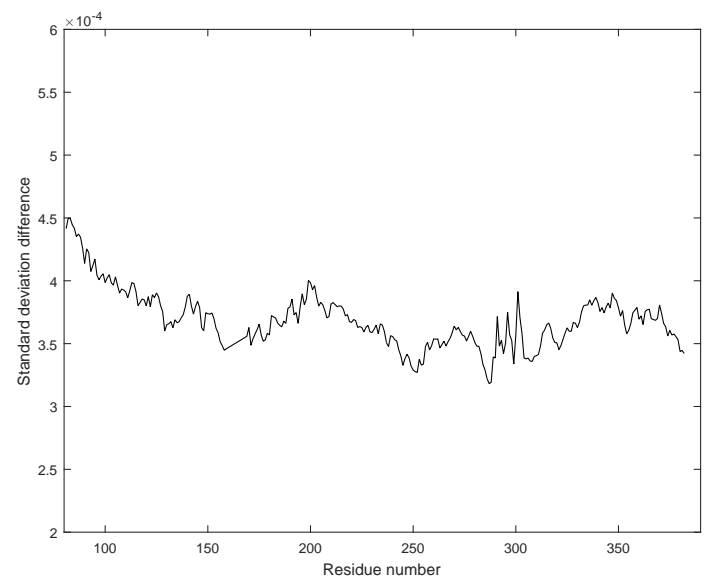

Figure 7: The standard deviation of $\mathrm{PhI}^{\mathrm{Pro}-}$ minus the standard deviation of $\mathrm{PhI}{ }^{\mathrm{Pro}+}$ plotted as a function of amino acid residue number for the average of the 7 peptides. The standard deviation maps are evaluated at the locations of the $\mathrm{C}_{\alpha}$ atoms. The locations of the $\mathrm{C}_{\alpha}$ atoms are taken from the combined x-ray and cryo EM structure of HK97. This combined structure includes $20 \%$ of the $\delta$-domain, which is disordered in the x-ray structure (Veesler et al., 2014), but does not include the inner $80 \%$ of the $\delta$-domain. 